\title{
"ll nostro destino splendido di viaggianti»
}

\author{
Die Rezeption der italienischen Avantgarde im Paris \\ des Surrealismus
}

\section{Die Ambivalenz der Moderne}

Am 8. Juli 2017 wurde die eritreische Hauptstadt zum Unesco Weltkulturerbe erklärt, "Asmara: eine modernistische afrikanische Stadt». ${ }^{1}$ Diese Widmung erfolgte bezeichnenderweise vor dem Hintergrund der von Afrika über das Mittelmeer nach Süditalien gelangenden Flüchtlingsströme sowie einer europäischen Politik, die auf diese Herausforderung immer wieder mit der Betonung von klaren nationalen Grenzziehungen reagiert. Eritrea war von 1890 bis 1941 italienische Kolonie; das koloniale Erbe Italiens, die hauptsächlich in den 1930er Jahren errichtete modernistische Architektur des Faschismus mit ihren rationalistischen Gebäuden, ist über die Jahrzehnte Teil der Identität des jungen Staates geworden, das «historische Asmara» fungierte als «Bezugspunkt im Bemühen um Unabhängigkeit» Eritreas von Äthiopien. ${ }^{2}$ Die Architekturtheoretiker Peter Volgger und Stefan Graf haben an der Universität Innsbruck das urbanistische Erbe Asmaras erforscht; die Ausstellung Asmara - The Sleeping Beauty zeigte die Ergebnisse des Projekts, das Architektur und Biopolitik in den Fokus nimmt. In Asmara machen Volgger und Graf ein «modernistisches Programm» aus, «welches auch ambivalente ideologische Momente der Moderne beinhaltet» und entgegen den postmodernen Tendenzen und der Globalisierung vom autoritären Staat gezielt für dessen «nation-building»-Projekt eingesetzt wird. ${ }^{3}$

Im Mittelpunkt der Aufnahme Asmaras in die Welterbeliste stand eine von dem italienischen Architekten Giuseppe Pettazzi im futuristischen Stil entworfene Tankstelle in Form eines startenden Flugzeugs, die 1938, in der Hochphase

1 Vgl. <https://www.unesco.de/kultur-und-natur/welterbe/welterbe-weltweit/asmara-eine-modernistische-stadt-afrikas-neue $>$ [29.10.2018].

2 Ebda.

3 Peter Volgger/Stefan Graf: Asmara - The Sleeping Beauty. Eine Ausstellung kuratiert von Stefan Graf und Peter Volgger. <https://aut.cc/ausstellungen/asmara-the-sleeping-beauty> [29.10.2018]; Vgl. auch Peter Volgger/Stefan Graf (Hg.): Architecture in Asmara - Colonial Origin and Postcolonial Experiences. Berlin: DOM publishers 2017.

Ә Open Access. (c) 2020 Eva-Tabea Meineke, publiziert von De Gruyter. (c) BY-NC-ND Dieses Werk ist lizenziert unter der Creative Commons Attribution-NonCommercial-NoDerivatives 4.0 Lizenz. https://doi.org/10.1515/9783110679366-003 
faschistischer Expansion, fertiggestellt worden war. ${ }^{4}$ Die afrikanischen Kolonien fungierten als «Experimentalräume» für die italienischen Avantgardisten, Asmara wurde beispielsweise als frühe «autogerechte Stadt» geplant. ${ }^{5}$ Die 〈beflügelte〉 Tankstelle, die als Fiat Tagliero bekannt ist, macht aus der eritreischen Hauptstadt bis heute einen futuristischen Ort, an dem sich Europa und Afrika, koloniale Vergangenheit und postkoloniale Gegenwart, künstlerischer Ausdruck, Technik und afrikanische Landschaft bzw. afrikanisches Licht auf ideale Weise verschränken. Der Kopf der Futuristen Filippo Tommaso Marinetti betont im Jahr der Errichtung der Fiat Tagliero in einem Vortrag an der Accademia d'Italia die Bedeutung Afrikas als Ursprung der (futuristischen) Künste und ihrer Poesie. ${ }^{6}$ Liegt die Schönheit des modernistischen Asmara womöglich in der Tatsache begründet, dass sich dort, trotz allen negativen Beigeschmacks der politischen Kolonisierung und des Faschismus, der Kreis des wechselseitigen Austausches Afrikas mit Europa wieder schließt, der Futurismus als Kunstform gewissermaßen in seinen $"$ Mutterschoß» ${ }^{7}$ zurückgekehrt ist, und daher die Identifikation der eritreischen Bevölkerung mit demselben funktioniert? Interessant ist es darüber hinaus zu beobachten, dass die modernistische Architektur dem jungen eritreischen Staat als Anker für die Herausbildung von Zusammenhalt und Nationalgefühl dient. Im Folgenden soll nachvollzogen werden, welche Rolle Migrationserfahrungen und die damit verbundene Auseinandersetzung mit anderen Kulturräumen, Kulturlandschaften und Sprachen im Hinblick auf die Herausbildung der ästhetischen Innovationen der frühen Avantgarde gespielt haben und wie diese künstlerische Arbeit an den Grenzen zum Nährboden für nationalistische Interessen werden konnte. Diese Ambivalenz der Moderne, die Volgger und Graf mit Blick auf Asmara betonen, gilt es bezüglich der italienischen Avantgarde in Europa zu beleuchten, die am Beispiel von Filippo Tommaso Marinetti und den Brüdern de Chirico, vor allem dem jüngeren Alberto Savinio, untersucht wird. Dabei sollen die Einflüsse der Italiener auf den Surrealismus in Paris im Vordergrund stehen, der sich seinerseits ambivalent gestaltet, indem er seine Ästhetik aus der Arbeit an den Grenzen gewinnt und sich trotzdem auch, wenn auch nicht im selben Maße wie der Futurismus, als nationale Bewegung begreift.

4 Vgl. Massimo Minella: Il sogno dell'ingegnere che disegnò le ali alla bellezza di Asmara. $<$ http://ricerca.repubblica.it/repubblica/archivio/repubblica/2017/07/17/il-sogno-dellingegnereche-disegno-le-ali-alla-bellezza-di-asmara21.html> [25.01.2018].

5 Peter Volgger/Stefan Graf: Asmara - The Sleeping Beauty.

6 Vgl. Pasquale A. Jannini: Introduzione. In: Filippo Tommaso Marinetti: Scritti francesi. Bd. 1. Mailand: Mondadori 1983, S. 7-30 (12).

7 Filippo Tommaso Marinetti wurde auf afrikanischem Boden, im ägyptischen Alexandria geboren. 


\section{Die italienische Avantgarde im Spannungsfeld von Migration und nationaler Anbindung: Filippo Tommaso Marinetti und die Brüder de Chirico}

Wichtige Einflüsse auf die Avantgarde in Paris, allen voran den Surrealismus, stammen von Italienern, die sich in privilegierten Migrationskontexten ${ }^{8} \mathrm{zu}$ Künstlern entwickelten und aus der erlebten Hybridität heraus neue Ideen formierten. Die italienische Avantgarde bildete sich bereits vor dem Ersten Weltkrieg heraus, man kann ihr eine Vorreiterfunktion in Europa ${ }^{9}$ zuschreiben und dies obwohl oder womöglich gerade weil - Italien sich mit seinem reichen kulturellen Erbe eigentlich dem «passatismo» verschrieben hatte. ${ }^{10}$ Die italienischen Künstler, wie Filippo Tommaso Marinetti oder die Brüder de Chirico, bewegten sich als dezidierte Grenzgänger bereits vor dem Ausbruch des Ersten Weltkriegs in Paris und gelangten 1909 mit dem futuristischen Manifest, das auf der Titelseite des Figaro erschien, und 1914 mit Andrea de Chiricos spektakulärem Auftritt am Klavier in den Räumen der Soirées de Paris, auf den Höhepunkt ihrer avantgardistischen Ausdruckskraft. ${ }^{11}$ Nach dem Krieg flossen ihre Impulse entscheidend in die Herausbildung des Surrealismus mit ein und beförderten die Entwicklung der ästhetischen Gestaltungsmittel dieser Bewegung. Paris fungierte dabei als Sammelbecken für die Ideen der italienischen Grenzgänger; sie fanden dort in abgerundeter Form - das surrealistische Manifest kommt lange nicht so provokant daher wie das futuristische - Einzug in die deutlich intellektueller ausgerichtete und auf Wissenschaftlichkeit zielende französische Avantgarde.

8 Anna Lipphardt verwendet in ihrer Theorie die Bezeichnungen «privilegierte Mobilität» und «mobile Hochqualifizierte». In: Dies.: Der Nomade als Theoriefigur, empirische Anrufung und Lifestyle-Emblem. Auf Spurensuche im Globalen Norden. In: APuZ 26/27 (2015). <http://www. bpb.de/apuz/208257/der-nomade-als-theoriefigur-empirische-anrufung-und-lifestyle-emblemauf-spurensuche-im-globalen-norden?p=all $>$ [25.01.2018].

9 Vgl. z.B. Walter Fähnders: Avantgarde und Moderne 1890-1933. Stuttgart: J.B. Metzler 1998, S. 132 und S. 193. Auch Gottfried Benn erklärt in seiner Marburger Rede «Probleme der Lyrik»: «Das Gründungsereignis der modernen Kunst in Europa war die Herausgabe des futuristischen Manifestes von Marinetti, das am 20. Februar 1909 in Paris im «Figaro〉 erschien». In: Ders.: Gesammelte Werke in vier Bänden. Bd. 1. Wiesbaden: Limes 1966, S. 494-532, hier S. 498.

10 Vgl. Sabine Schrader: Einleitung. In: Sabine Schrader/Barbara Tasser (Hg.): Futurismo al $100 \%$ - 100 \% Futurismus. Innsbruck: Innsbruck University Press 2012, S. 11-16, hier S. 12.

11 Vgl. dazu Eva-Tabea Meineke/Gesa zur Nieden: Kunst und Krieg bei Alberto Savinio. In: Zibaldone 57 (2014), S. 19-32. 
Italien ist auch das Land, in dem sich Anfang der 1920er Jahre erstmals der Faschismus herausbildete; diese Tatsache gilt in der historischen Forschung als Konsequenz der lange Zeit durch Fremdherrschaften und das Fehlen einer Nationalkultur bedingten Opferrolle des Landes. ${ }^{12}$ Das mit dem Risorgimento aufgekommene Nationalgefühl wurde in der Folge des Ersten Weltkriegs durch den Faschismus totalitaristisch instrumentalisiert; bereits der Futurismus um Marinetti hatte, womöglich ebenfalls aufgrund des historischen Unterlegenheitsgefühls der Italiener und in dezidierter Abgrenzung vom Decadentismo und seinem auf Verfeinerung der Sinne zielenden Ästhetizismus, jegliche Schwäche und die Verweiblichung mit Verachtung gestraft und sich stattdessen an der Kampfeslust und Verherrlichung des Krieges orientiert. Im Klima des durch den Ersten Weltkrieg geschürten Nationalismus wurden die Erfolge des Römischen Reichs, die schon im 19. Jahrhundert im Hinblick auf das Risorgimento eine wichtige Rolle gespielt hatten und beispielsweise von Leopardi beschworen wurden, ${ }^{13}$ von Mussolini genutzt, um ein neues römisches Impero $^{14} \mathrm{zu}$ formieren und sich seine eigene Machtposition in der Nachfolge der Imperatoren zu sichern. Im Hinblick auf die Kunst orientierte sich der Faschismus folglich zum einen an der Antike und dem Klassizismus; gleichzeitig schöpfte er aber auch die Funktionalität der Moderne aus und kollaborierte mit der Avantgarde. ${ }^{15}$ Es ist zu beobachten, dass beide künstlerischen Ausrichtungen, die neoklassische wie die moderne, im italienischen Kontext ambivalente Deutungen zulassen: Der Rückgriff auf die Antike kann zwar den imperialistischen Charakter des Römischen Reiches zum Ausdruck bringen und Italien im nationalistischen Sinne verherrlichen. Mit seiner Vielvölkerordnung, Mehrsprachigkeit und Multikulturalität verkörpert das Römische Reich aber auch ein Modell für ein vereintes Europa - auf diese letztere Weise wird die Antike im Werk der Brüder de Chirico und insbesondere des jüngeren Andrea de Chirico genutzt, der sich dabei vor allem auf den Polytheismus der Antike stützt. ${ }^{16}$ Analog

12 Vgl. u.a. Ruth Ben-Ghiat: Fascist Modernities: Italy 1922-1945. Berkeley: University of California Press 2001, S. 7.

13 Vgl. Giacomo Leopardi: All'Italia. In: Ders.: Canti. Herausgegeben von Niccolò Gallo/Cesare Garboli. Turin: Einaudi (1962) 1993, S. 3-10.

14 Vgl. George Talbot: Censorship in Fascist Italy 1922-43. Basingstoke: Palgrave Macmillan 2007, S. 2.

15 Vgl. Umberto Silva: Kunst und Ideologie des Faschismus. Frankfurt a.M.: S. Fischer 1975, S. 181-199.

16 Vgl. Peter Gahl: Die Fahrt des Argonauten. Das Werk Alberto Savinios von der «scrittura metafisica» zum «surrealismo archeologico». München: Wilhelm Fink 2003, S. 278. Gahl zitiert Savinio aus dem Stichwort «Germanesimo» der Nuova enciclopedia: «i Romani sono i creatori, per quanto inconsapevoli, di quella unione intellettuale di più popoli associati da comuni idee civili e morali che si esprime nel concetto Europa.» 
können auch die avantgardistischen Formen sowohl im aggressiven, kriegsverherrlichenden, die nationalen Fronten verschärfenden Sinne verstanden werden, als auch als Möglichkeit, durch die Arbeit an den Grenzen die Freiheit des Individuums zu befördern.

Filippo Tommaso Marinetti ist trotz seiner späteren Affinität zum Faschismus als Künstler national schwer zuzuordnen; erst Ende der 1960er Jahre ging der avantgardistische Marinetti mit der Veröffentlichung seiner Schriften in der Klassikerreihe der Meridiani von Seiten Luciano De Marias in die italienische Literaturgeschichte ein. ${ }^{17}$ Tatsächlich ist er ein "scrittore egizio-milanese-parigino» ${ }^{18}$ : Er wurde 1876 in der kolonialen Welt, auf afrikanischem Boden, im ägyptischen Alexandria geboren. Seine Eltern Enrico und Amalia Grolli hatten sich wenige Jahre zuvor dort niedergelassen, weil sein Vater zunächst als Zivilanwalt in den Geschäftsbüros der Gesellschaft für den Bau des Suezkanals tätig war, dann selbst mehrere Kanzleien eröffnete und später als Anwalt für den Khedive von Ägypten Muhammad Tawfiq Pascha bedeutenden Einfluss erlangte. ${ }^{19}$ Durch die ägyptischen Kontakte seines Vaters konnte Filippo Tommaso Marinetti später sein futuristisches Manifest auf der Titelseite des Pariser Figaro veröffentlichen. ${ }^{20}$ In Alexandria ging er auf das französischsprachige Jesuitenkolleg Saint-FrançoisXavier. Seine Mutter brachte ihm zu Hause die italienischen Klassiker nah, darunter Dante, aber auch D'Annunzio und machte ihn darüber hinaus mit Rousseau und Baudelaire im französischen Original vertraut. ${ }^{21}$ In Alexandria gründete Marinetti noch als Schüler die kleine Zeitschrift Papyrus - man beachte, dass der Name auf die antike Kultur des Mittelmeerraums zurückverweist, die Europa und Afrika verband. Die Kindheit und Jugend in Ägypten hat Marinettis Werk nachhaltig beeinflusst, er selbst formulierte dies einmal wie folgt: «Le pays africain a mordu sur mon tempérament». ${ }^{22}$ Seine erste Nahrungsaufnahme als Säugling, auf die er seine Vitalität zurückführt, habe er einer sudanesischen Amme $\mathrm{zu}$ verdanken, was er im autobiographischen Rückblick zu einer Er-

17 Vgl. Filippo Tommaso Marinetti: Teoria e invenzione futurista. Herausgegeben von Luciano de Maria. Mailand: Mondadori 1968.

18 Luigi Ballerini. Introduzione. In: Filippo Tommaso Marinetti: Mafarka il futurista. Mailand: Mondadori 2003, S. VII-XLVIII, hier S. XXII.

19 Vgl. Luigi Paglia: Filippo Tommaso Marinetti. In: Dizionario Biografico degli Italiani, Volume 70 (2008). <http://www.treccani.it/enciclopedia/filippo-tommaso-marinetti_(Dizionario-Biografico)/> [26.01.2018].

20 Luigi Ballerini: Introduzione. In: Filippo Tommaso Marinetti: Mafarka il futurista, S. VIII.

21 Vgl. Luigi Paglia: Filippo Tommaso Marinetti und Pasquale A. Jannini: Introduzione, S. 12.

22 Filippo Tommaso Marinetti zit. nach Pasquale A. Jannini, ebda. S. 14. 
weckungserfahrung stilisierte: «Cominciai in rosa e nero; pupo fiorente e sano fra le braccia e le mammelle color carbone coke della mia nutrice sudanese.» ${ }^{23}$

Fast achtzehnjährig verlässt Marinetti Ägypten, um in Paris sein baccalauréat zum Abschluss zu bringen. Danach kommt er, politisch und literarisch beeinflusst von dieser Pariser Erfahrung, nach Mailand. Bis 1909 schreibt Marinetti durchweg auf Französisch und dies nicht nur in der Literatur, sondern auch in seinen Briefen und Widmungen. Jannini vertritt sogar, dass Marinetti sich bis 1912 als französischer Schriftsteller begriff. ${ }^{24}$ Allerdings gesteht beispielsweise Marcel Raymond in seiner französischen Literaturgeschichte De Baudelaire au Surréalisme (1933 erstveröffentlicht und mehrmals wieder aufgelegt) dem Futurismus keine besondere Bedeutung im Kontext der Avantgarde zu; Marinetti wird darin mit keinem Wort als französischer Schriftsteller erwähnt. ${ }^{25}$ Erst 1976, zum hundertsten Geburtstag Marinettis, finden sich erste Anerkennungen von französischer Seite (z.B. von Pierre-Olivier Walzer), und Claude Bonnefoy bestätigt in seiner Rezension des von Giovanni Lista in der Reihe Poètes d'aujourd'hui in Paris (Seghers 1976) publizierten Portraits Marinetti: «Des initiateurs de la poésie moderne, Marinetti est le plus méconnu». ${ }^{26}$ Auch in Ägypten findet der 1905 von einem Journalisten in Kairo als ein «égyptien de grand talent et de grand avenir» bezeichnete Marinetti keinen Eingang in die Literatur- und Kulturgeschichte in französischer Sprache. ${ }^{27}$ Als Grenzgänger hatte er es schwer, in eine nationale Literaturgeschichtsschreibung einzugehen; im Kontext der sich zuspitzenden Nationalismen deklarierte er sich dann dezidiert als Italiener: «Venni a Milano bachelier es lettres [sic!], con una cultura francese, ma invincibilmente italiano, a dispetto di tutti i fascini parigini.» ${ }^{28}$

1908, ein Jahr vor der Publikation des futuristischen Manifests, hatte Émile Bernard Marinetti einen Dithyrambus gewidmet - und dies sicher nicht zufällig in der Form der antiken Gattung für Chorlyrik mit deren Rhythmus und Musikalität. Der Dithyrambus wurde in der sich an Paris orientierenden Zeitschrift Poesia veröffentlicht, die Marinetti 1905 selbst gegründet hatte, ${ }^{29}$ und fasst die hybride

23 Filippo Tommaso Marinetti: Scatole d'amore in conserva. Rom: Edizione d'Arte Fauno 1927, S. 8 .

24 Vgl. Pasquale A. Jannini: Introduzione, S. 7.

25 Vgl. Ebda., S. 9. Vgl. zum nationalistischen Charakter der Avantgarden auch Luigi Fontanella: Il Surrealismo italiano. Rom: Bulzoni 1983, S. 11.

26 Claude Bonnefoy zit. nach Pasquale A. Jannini: Introduzione, S. 10.

27 Vgl. Ebda., S. 11.

28 Filippo Tommaso Marinetti: Scatole d'amore in conserva, S. 13.

29 Vgl. Edoardo Costadura: Nascita dello scrittore uccello. In: Sergio Luzzatto/Gabriele Pedullà u. a. (Hg.): Atlante della letteratura italiana. Bd. III: Dal romanticismo a oggi. Turin: Einaudi 2012, 
nationale und kulturelle Identität des Kopfes der Futuristen mit Blick auf ihr vielfältiges künstlerisches Potential zusammen; Italien wird dabei die Musikalität zugeschrieben, Frankreich die Revolution und Ägypten das Licht: ${ }^{30}$

Trois Nations en vous ont leur Hôte présent:
L'Égypte et sa clarté dansante et son Désert,
L'Italie suave et ses Concerts
La France et son Ardeur de Révolte et de Sang;
Et vous êtes ainsi la belle Trilogie
De trois Forces en vous jetant leur énergie. ${ }^{31}$

Dass die Innovativität der hier zu diskutierenden italienischen Künstler markant von der Peripherie auf die sich in Paris entwickelnde Avantgarde wirkte, zeigen neben Marinetti insbesondere auch die Brüder de Chirico mit ihrer arte metafisica. ${ }^{32}$ Giorgio und Andrea de Chirico, der sich später Alberto Savinio nannte, ${ }^{33}$ wurden in Volos und Athen als Kinder von Evaristo de Chirico und Gemma Corvetto geboren. Da der Vater Evaristo als Direktor der Entreprise E. Chirico et Cie. am Bau der ersten Eisenbahnlinie Thessaliens beteiligt war, wechselte die Familie sehr häufig den Wohnort. ${ }^{34}$ Die Brüder lebten in Athen, München, Paris sowie in verschiedenen italienischen Städten und entwickelten ihre «metaphysische Poesie» im künstlerischen Austausch untereinander, der die Malerei, Musik und Literatur einbezog. ${ }^{35}$ Paola Italia zeichnet in ihrer umfangreichen Monographie Il pellegrino appassionato Savinios Entwicklung zum italienischen Schriftsteller im Zeitraum von 1915-1925 nach und betont, dass dieser nie einen wirklichen Schulabschluss erzielte, sondern vermutlich in Griechenland von seinem

S. 454-459, hier S. 454: «Decisamente protesa verso Parigi è la rivista 〈Poesia〉, che fonda e dirige fra il 1905 e il 1909 e nella quale andrà pubblicando i poeti simbolisti francesi e italiani.»

30 Afrikas Verbindung zum «Licht» bei Marinetti ist in Fondazione e Manifesto del Futurismo subtil durch den Vergleich der Futuristen mit «fari superbi» (S. 7) auszumachen, da der Begriff von «Pharos» stammt, dem ersten Leuchtturm der Geschichte, demjenigen Alexandrias.

31 Émile Bernard: À Marinetti. In: Poesia IV, 6 (Juli 1908), S. 6; Vgl. Luigi Ballerini: Introduzione, S. XV.

32 Vgl. zu den Einflüssen und Wechselwirkungen Italien - Frankreich im Umfeld des Surrealismus Verf.: Rivieras de l'irréel. Surrealismen in Italien und Frankreich. Würzburg: Königshausen \& Neumann 2019.

33 Vgl. zur Namensgebung Edoardo Costadura: Nascita dello scrittore-uccello, S. 454-459.

34 Vgl. Andrea Grewe: Melancholie der Moderne. Studien zur Poetik Alberto Savinios. Frankfurt a.M.: Vittorio Klostermann 2001, S. 11.

35 Vgl. Magdalena Holzhey/Gerd Roos: Giorgio de Chirico und Alberto Savinio. Eine Biografie der Dioskuren. In: Paolo Baldacci/Wieland Schmied (Hg.): Die andere Moderne. De Chirico Savinio. Ostfildern/Berlin: Hatje Cantz 2001, S. 25-43, hier S. 29. 
Vater die ersten literarischen Kenntnisse vermittelt bekam. 1903 erhielt Andrea de Chirico am Athener Konservatorium ein Diplom für Klavier und Komposition, bevor er ab 1906 in München bei Max Reger studierte. ${ }^{36}$ Dort wurde die Ausrichtung der Brüder auf die Vielfalt der Künste um eine Rezeption von Nietzsche, Schopenhauer und Böcklin in Verbindung mit Leopardi ${ }^{37}$ erweitert. In ihrer Mailänder Zeit 1909-1910 machten sie sich darüber hinaus mit weiteren italienischen Klassikern vertraut, vom Cinquecento bis ins Ottocento, ${ }^{38}$ und sie entwickelten gemeinsam eine neue Musikalität und Bildlichkeit.

Beide Brüder verfassten ihre literarischen Werke zunächst auf Französisch und integrierten das Italienische oder auch andere Sprachen und Dialekte, so z.B. Savinios Chants de la mi-mort (1914), Hermaphrodito (1919) oder de Chiricos Hebdomeros (1929). In Paris, wohin es die Brüder 1911 nach einander verschlug, ${ }^{39}$ erlebte Savinio «il suo simultaneo ingresso nella letteratura francese e nella letteratura italiana». ${ }^{40}$ Als sie 1915 aufgrund des Krieges, der sie auf ihre Nationalität zurückwarf, von Paris nach Ferrara übersiedelten, wiesen sie allerdings kaum Kenntnisse des Italienischen auf und situierten sich doch als «italienische> Künstler. Paola Italia, die in Florenz Archivmaterial von dieser Zeit im Hinblick auf Savinios «apprendistato letterario» untersucht hat, konstatiert, dass es sich bei Savinio um einen «scrittore italiano» handelt, "partito da una meno che scolastica conoscenza della lingua italiana, che inizialmente traduceva dal francese, con (dichiarata) 〈somma fatica〉.» ${ }^{41}$ Der Autor formierte sich in Ferrara, so Italia, indem er neben Handbüchern zu Grammatik und Stilistik auch Klassiker der italienischen Literatur studierte; besondere Bedeutung misst sie in diesem Zusammenhang dem Leopardi der Operette morali bei. ${ }^{42}$ Savinio schrieb in dieser Zeit sowohl auf internationaler Ebene für Tristan Tzaras Zeitschrift Dada als auch auf Italienisch für La Voce von Giuseppe de Robertis. ${ }^{43}$

Die Rezeption der Zeitgenossen fasst hauptsächlich Savinios «Fremdheit» und «Befremdlichkeit» ins Auge: «Non c’è scrittore italiano per gli italiani più «straniero» di Savinio», äußerte Leonardo Sciascia einmal. ${ }^{44}$ Papini wertet diese

36 Vgl. Paola Italia: Il pellegrino appassionato. Savinio scrittore 1915-1925. Palermo: Sellerio 2004, S. 27-28.

37 Vgl. Magdalena Holzhey/Gerd Roos: Giorgio de Chirico und Alberto Savinio, S. 29.

38 Vgl. Paola Italia: Il pellegrino appassionato, S. 28.

39 Andrea Grewe: Melancholie der Moderne, S. 13.

40 Edoardo Costadura: Nascita dello scrittore-uccello, S. 454.

41 Paola Italia: Il pellegrino appassionato, S. 14.

42 Vgl. ebda.

43 Edoardo Costadura: Nascita dello scrittore-uccello, S. 454.

44 Anmerkung Leonardo Sciascias zur «Introduzione» Héctor Bianciottis. In: Alberto Savinio: Souvenirs. Palermo: Sellerio ( $\left.{ }^{1} 1976\right)$ 1989, zit. nach Andrea Grewe: Melancholie der Moderne, S. 27. 
Tatsache positiv, denn er betont, dass Savinio der internationalen Avantgarde angehöre und daher eigentlich in Paris als dem «atelier dell'Europa» beheimatet sei. ${ }^{45}$ Andere, wie beispielsweise Bino Binazzi, schreiben dem Dichter, der die Sprachen so sehr mische, die Unfähigkeit zu, in der Sprache Dantes, einem stilistisch hochwertigen Italienisch zu schreiben oder heben, wie Francesco Meriano, nur seine patriotisch gefärbten Erzählungen aus der Zeit in Makedonien (von Juli 1917 bis Oktober 1918) hervor, wo er aufgrund seiner Griechischkenntnisse als Übersetzer zum Einsatz kam, sich jedoch umso mehr nach eigenen Kriegserlebnissen sehnte, die ihm wegen der Ausmusterung verwehrt blieben. ${ }^{46}$ In der nationalistisch und antimodern eingestellten italienischen Kultur der Zwischenkriegszeit wird Savinio aufgrund seiner europäischen Haltung und Angliederung an eine international ausgerichtete Moderne im Pariser Geist äußerst kritisch betrachtet, von manchen sogar verspottet; Marcello Carlino schreibt von «ostracismi e emarginazioni», mit denen der Dichter zu kämpfen hatte. ${ }^{47}$ Für die Zeitschrift La Ronda besprach Savinio in jenen Jahren die französische Literatur und fungierte als Vermittler einer europäisch ausgerichteten Kultur; nach dem Marsch auf Rom wurde das Erscheinen der Zeitschrift jedoch eingestellt, und auch Pirandellos Teatro d'Arte entwickelte sich zunehmend $\mathrm{zu}$ einer privaten Einrichtung, in der für Savinio kein Platz war. Sein «umorismo» und seine Ironie, die einen gewissen Pessimismus vertreten und den Ernst des Krieges nicht anzuerkennen scheinen, trafen in der Rezeption auf viel Unverständnis; mit dem sich in den 1920ern herausbildenden Faschismus, der auf Stärke und Durchsetzungsvermögen der Italiener zielte, erwiesen sie sich als unvereinbar. 1926 zog Savinio auf der Flucht vor der italienischen Autarkiepolitik erneut nach Paris um, wo er sieben Jahre verweilte und sich in den Kreisen der Avantgarde bewegte. ${ }^{48}$ Louis Aragon hatte den Künstler 1924 in seinem Manifest Une vague de rêves direkt adressiert und ihn sich zurück nach Paris gewünscht; er bezeugte dadurch dessen wichtigen Einfluss auf die Surrealisten.

45 Giovanni Papini: Alberto Savinio, zit. nach Andrea Grewe: Melancholie der Moderne, S. 28.

46 Vgl. Bino Binazzi: Dai «cabarets» di Parigi ai baraccamenti di Salonicco. Avventure liriche d'un caporale d'eccezione und Francesco Meriano: Umorismo futurista, zit. nach Andrea Grewe: Melancholie der Moderne, S. 28. Vgl. bezüglich der Kriegsbegeisterung Savinios Paola Italia, die sich auf dessen Korrespondenz mit Soffici beruft: Paola Italia: Il pellegrino appassionato, S. 70-71. Die physische Kriegsuntauglichkeit Savinios erinnert an Leopardi, bekannt als ein Intellektueller schlechter körperlicher Verfassung.

47 Vgl. Andrea Grewe: Melancholie der Moderne, S. 29; Marcello Carlino: Savinio, zit. ebda.

48 Vgl. Andrea Grewe: Melancholie der Moderne, S. 19; Peter Gahl: Die Fahrt des Argonauten, S. 366. 
Mon cher Savinio, abandonnez Rome et venez ici, poussant devant vous la charrette où sont entassés les corps des Niobides. Tout ce monde que j’ai dénombré vous attend. Sans doute qu'il va se passer de grandes choses. ${ }^{49}$

Bis zu seinem Tod 1952 blieb Savinio dennoch auf allen Seiten «ein Außenseiter, ein 〈Fremder»». ${ }^{50}$ Danach geriet der Künstler zunächst in Vergessenheit und wurde, ähnlich wie Marinetti, erst in den 1970er Jahren langsam wiederentdeckt. Seine Bücher wurden seit dieser Zeit neu aufgelegt, zunächst Infanzia di Nivasio Dolcemare 1973 und Hermaphrodito 1974, jeweils bei Einaudi. Mittlerweile sind fast alle Werke im Mailänder Adelphi Verlag erschienen. Seit 1973 wird Savinio auch in der Literaturwissenschaft diskutiert; Ugo Piscopo verfasste damals eine erste Monographie. ${ }^{51}$

\section{Die Arbeit an den Grenzen: ästhetische Ambivalenzen}

In Marinettis Werken sind die afrikanische Landschaft und die arabische Kultur im Zusammenhang mit der futuristischen Grenzüberschreitung präsent, wie beispielsweise sein in Afrika situierter Roman Mafarka il futurista (1909 auf Französisch, 1910 auf Italienisch erschienen) oder auch das futuristische Manifest zeigen. Das Manifesto del futurismo nimmt in der orientalisierend ausgestatteten Mailänder Wohnung Marinettis, seinem eigenen multikulturell besetzten «Heim», seinen Ausgang und ruft von dort zum ungezügelten Handeln und Schreiben im futuristischen Sinn auf, zum Vordringen an die «äußersten Grenzen» des Verstands - bemerkenswert ist hier schon die Nähe zur surrealistischen écriture automatique.

Avevamo vegliato tutta la notte - i miei amici ed io - sotto lampade di moschea dalle cupole di ottone traforato, stellate come le nostre anime, perché come queste irradiate dal chiuso fulgòre di un cuore elettrico. Avevamo lungamente calpestata su opulenti tappeti orientali la

49 Louis Aragon: Une vague de rêves. In: Ders.: Euvres poétiques complètes. Bd. 1. Herausgegeben von Olivier Barbarant. Paris: Gallimard 2007, S. 95.

50 Andrea Grewe: Melancholie der Moderne, S. 29-31. Vgl. auch Paola Italia: Savinio, Soffici e la politica culturale del fascismo nei primi anni Venti. «Il Nuovo Paese» e il «Corriere Italiano». In: Nuova Rivista di letteratura italiana (2000), S. 389-450. Italia bespricht in ihrem Aufsatz Savinios Verhalten im Kontext der faschistischen Zeitungen Anfang der 1920er Jahre und hebt seine Ironie und sein authentisches Künstlertum hervor.

51 Vgl. Peter Gahl: Die Fahrt des Argonauten, S. 9. 
nostra atavica accidia, discutendo davanti ai confini estremi della logica ed annerendo molta carta di frenetiche scritture..$^{52}$

$\mathrm{Zu}$ diesem auf den Surrealismus vorausdeutenden freiheitlichen Gestus des psychischen Automatismus gesellen sich im Sinne der ideologischen Ambivalenz des Futurismus Nationalismus und Kriegsverherrlichung. Wesentlich für Marinetti ist eine paradoxe Konstellation, die der für den Futurismus kennzeichnenden Explosivität zugrunde liegt. Diese ist entgegen der Theorie von Deleuze und Guattari, die «ihren Nomaden einst als subversive Figur in Opposition zum hegemonialen (französischen) Nationalstaat entworfen» haben, in einer Parallelführung von «Grenzüberschreitung, Subjektivierung, Flexibilität und Mobilität» ${ }^{53}$ auf der einen Seite und aggressivem Nationalismus auf der anderen zu verorten. Im Kontext der Avantgarde und mit Blick auf den Pariser Surrealismus der 1920er Jahre ist die künstlerische Leistung des Futurismus - trotz aller berechtigten Kritik, die Marinetti auszulösen vermag - nicht zu unterschätzen, und dies vor allem aufgrund des grenzüberschreitenden Potenzials, des Spiels mit Paradoxien und des bereits dem Surrealismus vorweggenommenen Einbezugs verdrängter Bereiche des Menschlichen. ${ }^{54}$ Von der Schriftstellerin Rachilde wurde Marinetti in einer Rezension des Mafarka im Mercure de France bezeichnenderweise mit dem den Surrealisten heiligen Lautréamont verglichen: «Mafarka m'a produit l'effet des Chants de Maldoror» ${ }^{55}$ Lautréamonts berühmte Definition der Schönheit - «Il est beau [...] comme la rencontre fortuite sur une table de dissection d'une machine à coudre et d'un parapluie! ${ }^{56}$ - bot den Surrealisten eine beispielhafte image surréaliste, die zuvor als «immaginazione senza fili» womöglich im Kern bereits von Marinetti ausgeschöpft worden war. ${ }^{57}$

So lassen sich auch noch im Manifeste du surréalisme aus dem Jahre 1924, fünfzehn Jahre später, deutliche Anklänge an Marinettis Manifesto del futurismo finden: Aus der futuristischen «immaginazione senza fili» wird die «image sur-

52 Filippo Tommaso Marinetti: Fondazione e Manifesto del Futurismo. In: ders.: Teoria e invenzione futurista, S. 7-13, hier S. 7. Von der Verfasserin markiert sind die orientalisierenden Elemente sowie die Antizipation der automatischen Schreibweise des Surrealismus.

53 Vgl. Anna Lipphardt: Der Nomade als Theoriefigur.

54 Im Hinblick auf die Grenzüberschreitung zu ergänzen wäre überdies Marinettis Arbeit an den Geschlechtergrenzen, die vor allem auch im Mafarka abzulesen ist, vgl. dazu Elisabeth Tiller: Futuristische Männlichkeiten. In: Zibaldone 57 (2014), S. 77-90.

55 Vgl. Luigi Ballerini: Introduzione, S. XXI.

56 Lautréamont: Chants de Maldoror. Chant sixième. I [3]. In: Ders.: Germain Nouveau. Euvres complètes. Paris: Gallimard 1970, S. 224-225.

57 Vgl. Filippo Tommaso Marinetti: Manifesto tecnico della letteratura futurista. In: Filippo Tommaso Marinetti: Teoria e invenzione futurista, S. 46-54, hier S. 53. 
réaliste». In seiner «Introduction au Discours sur le peu de réalité» schreibt Breton im selben Jahr auch von «imagination sans fil»; ${ }^{58}$ dabei betont er «Sans fil, voici une locution qui a pris place trop récemment dans notre vocabulaire ${ }^{59}$. Weiterhin kann man die Schnelligkeit («vitesse» ${ }^{60}$ ), die Breton zufolge der écriture automatique zugrunde liegt und die surrealistischen Bilder unbewusst miteinander verkettet, auf Marinettis Ästhetik der Geschwindigkeit, seine «frenetiche scritture», «catena delle analogie» und die Vorstellung von «strette reti d'immagini o analogie» zurückführen. ${ }^{61}$ Beide ästhetischen Prinzipien des Surrealismus, die écriture automatique und die image surréaliste, sind folglich bereits im Kern bei Marinetti angelegt. Weitere Beispiele der Einflussnahme futuristischer Ästhetik auf den Surrealismus lassen sich insbesondere am Einbezug der Reklame, an akustischen Phänomenen und an der Ästhetik des Metallischen und der Phosphoreszenz festmachen. ${ }^{62}$

Der Aporie der Avantgarde entspricht der Futurismus, neben seiner Parallelführung von Grenzüberschreitung/Migration und Nationalismus, indem er trotz der radikalen Abwendung vom Vergangenen und dem verschrienen «passatismo» letztlich nicht ohne eine, wenn auch subtile, Anbindung an Gewesenes, auch Persönlich-Biographisches auskommt. So steht z.B. die von Marinetti im Manifest deklarierte Ablehnung der Nike von Samothrake aus dem Louvre - «un automobile ruggente, che sembra correre sulla mitraglia, è più bello della Vittoria di Samotracia» ${ }^{63}$ - im Widerspruch sowohl zur futuristischen Verherrlichung des Sieges (vittoria) als auch zur Beflügelung, die, der Tradition entlehnt, z. B. auch in der Tankstelle von Asmara zutage tritt. Im Manifesto tecnico della letteratura

58 André Breton: Introduction au Discours sur le peu de réalité. In: Ders.: Euvres complètes. Bd. 2. Herausgegeben von Marguerite Bonnet. Paris: Gallimard 1992, S. 265-280, hier S. 265.

59 Ebda.

60 André Breton: Manifeste du surréalisme. In: Ders: Euvres complètes. Bd. 1. Herausgegeben von Marguerite Bonnet. Paris: Gallimard 1988, S. 307-346, hier S. 326, und S. 332: «Écrivez vite sans sujet préconçu, assez vite pour ne pas retenir et ne pas être tenté de vous relire».

61 Filippo Tommaso Marinetti: Manifesto tecnico della letteratura futurista, S. 49.

62 Vgl. zu den Einflüssen des Futurismus auf den Surrealismus bzw. der Wirkung des Futurismus nach Paris weiterhin Rossana Jemma: La réception immédiate du Manifeste de fondation du Futurisme de Marinetti en France. In: Mariella Colin: Lettres italiennes en France. Caen: Presses Universitaires de Caen 2005, S. 145-161; Noëmi Blumenkranz-Onimus: Le pré-surréalisme des futuristes italiens. In: Sandro Briosi/Henk Hillenaar (Hg.): Vitalité et contradictions de l'avantgarde. Italie-France 1909-1924. Paris: José Corti 1988, S. 27-37; Giovanni Lista: Marinetti et le surréalisme. In: Surréalisme/Surrealismo, Quaderni del Novecento Francese 2 (1974), S. 121-149; Beatrice Sica: La poesia italiana e il surrealismo. In: Dies.: Poesia surrealista italiana. Genua: San Marco dei Giustiniani 2007, S. 9-49, hier S. 12-21 und Luigi Fontanella: Il surrealismo italiano. Ricerche e letture. Rom: Bulzoni 1983.

63 Filippo Tommaso Marinetti: Fondazione e Manifesto del Futurismo, S. 10-12. 
futurista, dessen Grundprinzipien Marinetti in einem Flugzeug sitzend ersinnt, verwendet der Kopf der Futuristen selbst das Bild der «Beflügelung〉 im Hinblick auf die Freiheitlichkeit seiner Poetik der «immaginazione senza fili» und der "parole in libertà» und spielt den Höhenflug gegen das ansonsten so verehrte Automobil aus:

Perché servirsi ancora di quattro ruote esasperate che s'annoiano, dal momento che possiamo staccarci dal suolo? Liberazione delle parole, ali spiegate dell'immaginazione, sintesi analogica della terra abbracciata da un solo sguardo e raccolta tutta intera in parole essenziali. $^{64}$

Trotz des vordergründigen Kampfes gegen alles Vergangene und seiner Vorliebe für die Industrialisierung und Technisierung gelang es dem Futurismus also eigentlich nicht gänzlich, auf eine Anbindung an Tradition und Geschichte zu verzichten: «Pur essendo nato ad Alessandria d'Egitto, io mi sento legato alla foresta di camini di Milano e al suo vecchio Duomo» ${ }^{65}$, erklärte Marinetti im Hinblick auf die italienische Metropole der Moderne Mailand. Und sein Roman Mafarka il futurista gliedert sich mit der Herrschaft Mafarkas über Afrika zwar in den kolonialen Kontext des Italiens der Zeit ein, ruft aber auch - dies unterstreicht Luigi Ballerini in seiner Einleitung zum Roman - die Geschichte des Römischen Reichs, der Latinität und des Mare Nostrum auf, was die nationalen Grenzen wiederum verwischen lässt. ${ }^{66}$ Bereits ein Jahrhundert zuvor hatte Giacomo Leopardi mit seinem Gedicht All'Italia, damals im Ausblick auf das Risorgimento, die antike Geschichte ins Bewusstsein gerufen, die «venturose e care e benedette / [...] antiche età che a morte / per la patria correan le genti a squadre». ${ }^{67}$ Der berühmte italienische Dichter des Pessimismus, an den sich Marinetti in politischer Dimension anlehnt, wird im futuristischen Fokus aufgrund seiner revolutionären, zum Kampf aufrufenden Haltung zum «maestro d'ottimismo» umstilisiert. ${ }^{68}$ Mit Versen wie den folgenden aus dem Gedicht Canto notturno di un pastore errante dell'Asia (1829-30, 1831 erstveröffentlicht), die Ballerini seiner Einleitung des Mafarka voranstellt, nimmt Leopardi die futuristi-

64 Filippo Tommaso Marinetti: Manifesto tecnico della letteratura futurista, S. 53.

65 Filippo Tommaso Marinetti zit. nach Luigi Ballerini: Introduzione, S. XV-XVI.

66 Vgl. ebda., S. XVII.

67 Giacomo Leopardi: All'Italia, S. 7.

68 Vgl. Filippo Tommaso Marinettis Schrift zu Leopardis 100. Todestag 1938: «Leopardi, maestro d'ottimismo». Vgl. zum Verhältnis Marinetti - Leopardi auch Emanuele La Rosa: Leopardi, Marinetti, Futurismo e futurismi, Un rapporto infinito. In: Barbara Kuhn/Michael Schwarze (Hg.): Leopardis Bilder. Immagini e immaginazione oder: Reflexionen von Bild und Bildlichkeit. Tübingen: Narr Francke Attempto 2019, S. 171-185. 
sche Energie, den 〈beflügelten〉 Aufschwung (zu den Sternen) und mit dem Donner auch wichtiges akustisches Material der Kunst Marinettis vorweg.

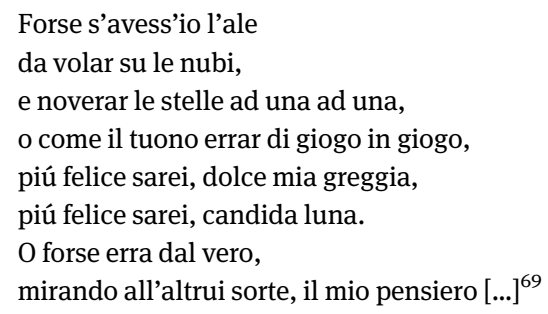

Die Brüder de Chirico entwickelten analog zu Marinetti ebenfalls bereits vor dem Ersten Weltkrieg eine avantgardistische Kunst, die Breton und die französischen Surrealisten nachhaltig beeindruckte. Dies formulierte der Wortführer der surrealistischen Bewegung 1940 im Rückblick seiner Anthologie de l'humour noir, wo er die neue, neben der Malerei die Musik einbeziehende Universalsprache der Brüder, die er als den Urgrund der Moderne ausmacht, hervorhebt; besonders betont er dabei die Leistung des bis dato vernachlässigten Alberto Savinio und vor allem seinen Auftritt vor den Vertretern der Pariser Avantgarde kurz vor Ausbruch des Krieges, bei dem er die Chants de la mi-mort am Klavier inszenierte.

Tout le mythe moderne encore en formation s'appuie à son origine sur les deux œuvres dans leur esprit presque indiscernables, d'Alberto Savinio et de son frère Giorgio de Chirico, œuvres qui atteignent leur point culminant à la veille de la guerre de 1914. Les ressources du visuel et de l'auditif se trouvent par eux simultanément mises à contribution pour la création d'un langage symbolique, concret, universellement intelligible du fait qu'il prétend rendre compte au plus haut degré de la réalité spécifique de l'époque [...]. ${ }^{70}$

Savinios Frühwerk Hermaphrodito (1918), in dem er seine gesamte Poetik angelegt sieht, ${ }^{71}$ zeugt, der Avantgarde entsprechend, auf allen Ebenen von Hybridität, indem es Gattungen, Sprachen - Italienisch, Französisch und andere lebende und tote Sprachen sowie Dialekte des Italienischen - und Register mischt und

69 Giacomo Leopardi: Canto notturno di un pastore errante dell'Asia. In: Ders.: Canti, S. 187-194, hier S. 194.

70 André Breton: Anthologie de l'humour noir. In: Ders.: Euvres complètes. Bd. 2, S. 1122-1126, hier S. 1122, Markierung von der Verfasserin.

71 Vgl. Peter Gahl: Die Fahrt des Argonauten, S. 14, und weiterhin, zu Savinios Frühwerk (Chants de la mi-mort und Hermaphrodito) die Monographie von Marco Sabbatini: L'argonauta, l'anatomico, il funambolo. Alberto Savinio dai Chants de la mi-mort a Hermaphrodito. Rom: Salerno 1997. 
darüber hinaus auch typographische Besonderheiten einbezieht. ${ }^{72}$ Der Titel impliziert ein der antiken Mythologie entlehntes sexuelles Mischwesen mit männlichen und weiblichen Geschlechtsorganen, von dem Ovids Metamorphosen, aber auch die von den Surrealisten so bewunderten Chants de Maldoror Lautréamonts berichten; die Episode aus den Chants zum Hermaphroditen wurde kurz vor dem Krieg erstmalig in Italien, in der Zeitschrift Lacerba veröffentlicht und war Savinio vermutlich bekannt. ${ }^{73}$ Mit Hermaphrodito antizipierte der Grenzgänger bereits das Prinzip der image surréaliste, Widersprüche $\mathrm{zu}$ vereinen. Giovanni Papini beschreibt das hybride Werk als «emporio levantino, un bazar di tappeti e d'ottoni, dove soltanto il padrone può ritrovarsi» ${ }^{74}$. Die orientalisierenden Bezüge, die Papini herstellt, die Teppiche und Gegenstände aus Messing, weisen interessanterweise Parallelen zu Marinettis Mailänder Wohnung auf, von der der Futurismus mitsamt dem Gestus des Automatismus seinen Ausgang nahm. Auch Savinio antizipierte bereits die wesentlichen ästhetischen Verfahrensweisen des Surrealismus. Seinem künstlerischen Auge zu verdanken hatte er die Hybridität der image surréaliste - bereits in der pittura metafisica seines Bruders wurden Gegenstände aus ihrem gewohnten Kontext herausgelöst und ganz neu kombiniert, so dass sie überraschende "Beziehungen» eingehen konnten; ${ }^{75}$ durch seine Nähe zur Musik tendierte er zu einer rhythmisch gestalteten écriture. Dabei steht bei ihm, im Gegensatz zu Marinetti und Breton, allerdings nicht die Geschwindigkeit im Vordergrund. Die Verkettung der Bilder, akustische Phänomene und die Ästhetik des Metalls und des Mechanischen als Ergänzung zum Natürlichen spielen jedoch analog zu Marinetti auch bei Savinio eine wesentliche Rolle, beispielsweise in der von ihm selbst auf 1919 datierten Tragedia dell'infanzia. ${ }^{76}$ Reklame, fabrikmäßig produzierte Gegenstände oder auch Abfälle der Konsumgesellschaft, die den modernen Alltag bestimmen, sind hingegen bei ihm, im Gegensatz zu Marinetti, kaum ein Thema. Mit Kunstwerken wie dem Titelblatt der Scatole d'amore in conserva (1927) Marinettis, von Carlo Petrucci gestaltet, das eine geöffnete Konservendose mit originell gestaltetem Etikett vor gelbem Hinter-

72 Vgl. Peter Gahl: Die Fahrt des Argonauten, S. 96.

73 Vgl. ebda., S. 99.

74 Giovanni Papini, Rezension zum Hermaphrodito In: «Il Resto del Carlino», zit. nach Peter Gahl: Die Fahrt des Argonauten, S. 96. Auch Edoardo Costadura erwähnt dieses Zitat und stellt es in den Zusammenhang der Herkunft der Eltern de Chirico: Der Vater Evaristo entstammte einer Diplomatenfamilie aus Ragusa in Dalmatien und zog dann nach Istanbul; die Mutter Gemma Cervetto gehörte einer Händlerfamilie aus Smyrna an, die entfernten italienischen Ursprungs war. Vgl. Nascita dello scrittore-uccello, S. 455.

75 Vgl. Peter Gahl: Die Fahrt des Argonauten, S. 37.

76 Vgl. Alberto Savinio: Tragedia dell'infanzia, Mailand: Adelphi 2001, S. 130. 
grund zeigt, deutet der Futurismus bereits auf die Popart voraus und legt durch seine Orientierung an Konsumgegenständen und die innovative Graphik den Grund für das italienische Werbedesign bis heute. Die futuristische Kunst löst sich in derartiger Gestaltung dezidiert von der antiken Vergangenheit Italiens. ${ }^{77}$

Savinio hingegen wird nach dem Krieg eine Hinwendung zu klassizistischen Formen zugeschrieben, die als Möglichkeit ausgelegt wird, sich besser im italienischen Kontext des ritorno all'ordine $\mathrm{zu}$ etablieren. ${ }^{78}$ Die Forschung vertritt darüber hinaus auch die These von Savinios «spezifischer Synthese» aus der künstlerischen Avantgarde und einem klassischen Kultur- und Geschichtsbewusstsein; Savinio sei, im Gegensatz zu seinem Bruder, der metaphysischen Kunst stets verschrieben gewesen. ${ }^{79}$ Anders als Marinetti bezog Savinio jedenfalls die eigene biographische Vergangenheit und Elemente der Geschichte bis zurück in die mythische Antike in seine Kunst ein; seine Art und Weise des Umgangs mit der Geschichte ist derjenigen des Surrealismus vergleichbar, der ebenfalls durch 〈Spiegel〉 Begebenheiten der Vergangenheit in der Gegenwart auferstehen lässt und damit dem Subjekt, das biographisch dem Autor entspricht, zu einer erweiterten Wahrnehmung verhilft. ${ }^{80}$ Die produktive Ambivalenz der Moderne zwischen Grenzgängertum und nationaler Zugehörigkeit bzw. der Einschreibung in eine kulturelle Tradition, die Marinetti mit seinem radikalen Kampf gegen alles Vergangene und seine politische Ausrichtung am Faschismus gefährdete, findet sich bei Savinio bewusster ausgeschöpft; er übte durch diese seine Arbeit an den nationalen, zeitlichen und medialen Grenzen einen entscheidenden Einfluss auf den sich in Paris entwickelnden Surrealismus aus. Die Komplexität seiner Kunst, die einen tragenden europäischen Geist zu vermitteln vermag, bedingt allerdings eine sperrige Rezeption, die letztlich nur wenigen vorbehalten bleibt. Leichter zugänglich ist bis heute der Futurismus, der zum einen die Alltagskultur stärker einbezieht und sich zum anderen deutlicher an Italien anbinden lässt.

77 Vgl. Filippo Tommaso Marinetti: Scatole d'amore in conserva. Vgl. im Hinblick auf die Bedeutung des Futurismus für die Werbung auch die Entwürfe für Campari von Fortunato Depero.

78 Vgl. u.a. Edoardo Costadura: Nascita dello scrittore-uccello, S. 455.

79 Vgl. Peter Gahl: Die Fahrt des Argonauten, S. 18 und S. 367.

80 Vgl. z.B. Eva-Tabea Meineke: «[C]e chèvrefeuille tremblant». Der französische Surrealismus und das Mittelalter. In: Anna Isabell Wörsdörfer/Kirsten Dickhaut u.a. (Hg.): Sur les chemins de l'amititié. Beiträge zur französischen Literaturgeschichte. Freundesgabe für Dietmar Rieger. Wiesbaden: Harrassowitz 2017, S. 113-124. 


\title{
IV Die italienische Avantgarde und der französische Surrealismus
}

\author{
«Mes poisons sont les vôtres: voici l'amour, la force, la vitesse.» \\ Louis Aragon, Paysan de Paris («Discours de l’Imagination»)
}

Anhand der Dynamik des Autounfalls soll exemplarisch aufgezeigt werden, inwiefern der Automatismus, der verdrängte Bereiche des menschlichen Seins hervorholt und neu integriert, in Marinettis Manifesto del futurismo bereits vorhanden ist und bei Louis Aragon und André Breton in die jeweils auf eigene Weise praktizierte écriture automatique mündet. Der thesenhafte Forderungskatalog des futuristischen Manifests wird in Marinettis Text Fondazione e Manifesto del Futurismo als fruchtbares Ergebnis eines Unfalls präsentiert, der auf eine rasende Autofahrt folgt. Das Automobil, das er zuletzt in den Graben steuert, symbolisiert den getriebenen Menschen, der sich ganz dem Wahnsinn («pazzia»), den aggressiv-animalischen Trieben («belve», «leoni», «pescecane»), dem Todestrieb («inseguivamo la Morte», «la Morte, addomesticata») und dem Sexualtrieb («nascita del Centauro», «Regina crudele») verschrieben hat. Im übertragenen Sinne geht es hier um eine automatische Schreibweise, die fern der Ratio zur freien Entfaltung des radikalen Ausdrucks gelangt. Das Ergebnis werden die «parole in libertà» sein. Im nach der «immaginazione senza fili» verfassten Gründungstext des Futurismus sind die Bilder für die verschiedenen Triebe auf poetische Weise miteinander verwoben. Mit der Bruchlandung im Graben wird beispielsweise eine Neugeburt, eine Rückkehr in den Uterus («materno fossato»), bzw. an die Mutterbrust verbunden, ein Motiv, das auch die Surrealisten später ausschöpften. Der schwarze Schlamm, den er schmeckt, erinnert Marinetti in diesem Bildzusammenhang an die Milch, die ihm seine sudanesische Amme verabreicht hatte; er wird an seine afrikanischen Ursprünge zurückbefördert und kann sich dadurch wieder aufrichten; das glühende Eisen des Automobils überträgt sich auf sein Herz in Form von durchströmender Freude.

\footnotetext{
Tagliai corto, e pel disgusto, mi scaraventai colle ruote all'aria in un fossato... Oh! materno fossato, quasi pieno di un'acqua fangosa! Bel fossato d'officina! Io gustai avidamente la tua melma fortificante, che mi ricordò la santa mammella nera della mia nutrice sudanese... Quando mi sollevai - cencio sozzo e puzzolente - di sotto la macchina capovolta, io mi sentii attraversare il cuore, deliziosamente dal ferro arroventato della gioia! ${ }^{81}$
}

81 Filippo Tommaso Marinetti: Fondazione e Manifesto del Futurismo, S. 9. 
Der Text ist von Bildern durchzogen, die Natur und Technik miteinander verbinden, beispielsweise wird das Automobil zum Haifisch («pescecane»), es hat Schuppen und Flossen («squame» und «pinne») und der Schlamm wird zu «Metallmüll» und «himmlischem Ruß» («scorie metalliche» und «fuliggini celesti»). Mit diesen surrealistischen Bildern avant la lettre antizipiert Marinetti Savinios Darstellungen beispielsweise in der Tragedia dell'infanzia (vom Autor selbst auf 1919 datiert), die insbesondere im zentralen Kapitel Nel fondo del mare vorkommen und wiederum Louis Aragons Unterwasserszene im Paysan de Paris (1924/25 verfasst, 1926 erschienen) nachhaltig beeinflussten. In beiden Texten impliziert die Meeresmotivik eine Rückkehr in den Uterus, die von erotischer Initiation begleitet wird; das Metallische taucht in Savinios und Aragons Bildwelt, wie bei Marinetti, in Verbindung mit natürlichen Elementen auf, ergänzt werden darüber hinaus die Mythen: Bei Savinio handelt es sich auf dem Meeresboden um eine antike Göttin, die ein metallisches Skelett, «il gioco delle cerniere e degli anelli», aber auch Langustenaugen und eine "voce di sirena» besitzt, ${ }^{82}$ bei Aragon zeigt die Meereswelt des in grünliches Licht getauchten Pariser Spazierstockladen «une sirène au sens le plus conventionnel du mot», «qui se terminait par une robe d'acier ou d'écaille, ou peut-être de pétales de roses». ${ }^{83}$

Von den Pariser Surrealisten hat in der Prosa insbesondere Louis Aragon die bedingungslose Hingabe an die écriture automatique als grenzüberschreitendes ästhetisches Prinzip praktiziert. Bereits in seinem parallel $\mathrm{zu}$ Breton verfassten Manifest Une vague de rêves (1924) betont er die Notwendigkeit von Verlust und Scheitern im Hinblick auf die Surrealitätserfahrung: Sein Mythos ist Phaeton, der Jüngling, der am Himmel die Kontrolle über den Sonnenwagen verliert und abstürzt. ${ }^{84}$ Bezeichnenderweise nutzt Aragon - darin entspricht er Savinio, dem er in Une vague de rêves seine Verehrung kundtut - den Rückgriff auf die griechische Mythologie und geht mit dem Aufschwung in die Lüfte über das moderne Automobil hinaus. Auf den Höhenflug folgt dann der Absturz in die Tiefe.

Par quel hasard ne lit-on pas sur un monument de nos villes: À Phaéton, l'humanité reconnaissante? Qu'importe? Il a eu le goût du vertige et il est tombé! ${ }^{85}$

82 Alberto Savinio: Tragedia dell'infanzia, S. 113-114.

83 Louis Aragon: Le paysan de Paris. Paris: Gallimard (1926) 1953, S. 31.

84 Vgl. David Nelting/Isabel von Ehrlich: Phaëton. In: Maria Moog-Grünewald (Hg.): Mythenrezeption: die antike Mythologie in Literatur. Musik und Kunst von den Anfängen bis zur Gegenwart. Stuttgart: J.B. Metzler 2008, S. 571-577, hier S. 571. Vgl. im Zusammenhang der futuristischsurrealistischen Mythen die Modellbezeichnung «Phaeton» für eine Luxuslimousine bei VW.

85 Louis Aragon: Une vague de rêves, S. 96. 
Im Paysan de Paris ist ebenfalls die Rede vom Schwindel («vertige») und von der Geschwindigkeit («frénesie»), die im Surrealismus eine bedeutende Funktion übernehmen:

[...] vous allez enfin vous perdre, voici la machine à chavirer l'esprit. [...] un vertige de plus est donné à l'homme: le Surréalisme, fils de la frénésie et de l'ombre. ${ }^{86}$

Nur bei André Breton ist die Hingabe an den Automatismus nicht einem Selbstmord vergleichbar. ${ }^{87}$ Der Surrealistenanführer beharrt auf dem «instinct de conservation» und folgt nicht dem Vorschlag Nadjas, mit dem Wagen gegen einen Baum zu fahren: «Inutile d'ajouter que je n'accédai pas à ce désir». ${ }^{88} \mathrm{Er}$ strukturiert seinen Roman nach der image surréaliste und seine écriture kommt weniger fließend, weniger musikalisch-wellenartig daher als diejenige Aragons. Auch im Hinblick auf seine surrealistische Bewegung hält er an einer klaren, auch hierarchischen Struktur fest. Nur in Gedanken gibt er - wenn überhaupt - die Kontrolle ab und findet sich dann oft mit verbundenen Augen am Steuer des rasenden Automobils wieder: «Idéalement au moins je me retrouve souvent, les yeux bandés, au volant de cette voiture sauvage.» ${ }^{89}$

Die rasende Autofahrt und der Unfall als Schockmoment symbolisieren jeweils die écriture der Autoren, die - basierend auf dem Automatismus - einen je eigenen Rhythmus und eine eigene Musikalität offenbart.

\section{Zwischen Nation(alismus) und Migration: Futurismus heute}

In seiner politischen Ausrichtung hatte sich der gewaltbereite Futurismus dem Faschismus, übersteigertem Nationalismus und Totalitarismus verschrieben. Als Kunstform basierte er jedoch auch auf Grenzüberschreitungen national-kultureller, historisch-zeitlicher und medialer Art; er leistete Wesentliches für die Ästhetik der europäischen Avantgarde und hat den französischen Surrealismus nachhaltig beeinflusst. Avantgarde ist grundsätzlich ein in sich widersprüchliches Phänomen, darin liegt ihr explosives Potenzial; im italienischen Futurismus ist diese Widersprüchlichkeit besonders scharf wahrnehmbar. Dass sich hinter der offensi-

86 Louis Aragon: Le paysan de Paris, S. 81.

87 Vgl. Rita Bischof: Teleskopagen, wahlweise. Der literarische Surrealismus und das Bild. Frankfurt a.M.: Vittorio Klostermann 2001, S. 75-76.

88 André Breton: Nadja. In: Ders.: Euvres complètes. Bd. 1, S. 748.

89 Ebda. 
ven Fassade der kriegs- und kampfeslüsternen Futuristen - nicht umsonst ist der Avantgarde-Begriff selbst dem militärischen Kontext entlehnt - auch auf Migration basierende kulturelle Vernetzungen verbergen, zeigen die Zusammenhänge, in denen heute noch futuristischer Kunst ein wichtiger Stellenwert zukommt. Neben der eingangs bereits erwähnten Auszeichnung der eritreischen Hauptstadt Asmara als Modernist City of Africa, ließe sich auch das folgende Beispiel betrachten: Auf der Expo in Mailand 2015 zum Thema «Nutrire il pianeta, energia per la vita» wurde im italienischen Pavillon, im Empfangssaal für die Delegationen der Länder, Giacomo Ballas imposantes Ölgemälde auf Tapisserie «Genio futurista» ausgestellt. $^{90}$ In den italienischen Farben rot, weiß, grün vor einem hellblau bis blauen Hintergrund gehalten, kann man in der Mitte der prismatischen Anordnung die schematische Figur eines Menschen erkennen, dessen Kopf ein Stern darstellt und dessen gestreckte Arme ein «M» als Initiale Marinettis bilden. Das Werk zeigt mit seinen Formen und Farben die dynamischen Beziehungen des Universums und deutet in seiner malerischen Komposition auch auf klangliche Dimensionen hin («forme-rumore»). Es war erstmals 1925 auf der Weltausstellung in Paris gezeigt worden. Im Kontext der Mailänder Expo 2015 und der Wirtschaftskrise, die aktuell Italiens Position in Europa gefährdet, fungiert die futuristische Kunst erneut als Symbol für die Stärke des Landes und seine Ausrichtung auf die Zukunft, die «forza del Sistema Paese» wird im offiziellen Bericht über die Expo besonders betont:

La storia della realizzazione di Expo Milano 2015 è una best practice di come l'Italia e gli italiani, facendo squadra, siano capaci di realizzare qualunque progetto complesso. E' un esempio della forza del Sistema Paese, di quel conubio tra pubblico e privato che trova slancio nelle sue tante eccellenze e nell'apertura verso il mondo. ${ }^{11}$

Nimmt man jedoch die migratorischen Impulse ernst, die insbesondere durch den Grenzgänger Filippo Tommaso Marinetti selbst gesetzt wurden, so entspricht das Gemälde auch der «apertura verso il mondo» und erlaubt einen Ausblick auf größere Dynamiken und Vernetzungen sowie einen Rückbezug auf die Ursprünge des Futurismus in Afrika und auf den Mittelmeerraum als Wiege der europäischen Kultur. Vor dem Hintergrund der Krise des Landes, der rechtspopulistischen Tendenzen in der Politik und der Flut von Flüchtlingen aus ehemaligen afrikanischen Kolonien sendet die Avantgarde in der Gegenwart gerade durch ihre

90 Vgl. u.a.: Unbekannte/r Autor/in: Expo, dopo 90 anni in mostra il «Genio futurista» di Balla. <http://milano.corriere.it/notizie/cronaca/15_marzo_02/expo-in-mostra-genio-futurista-balla5e961592-c0f3-11e4-b2c9-4738a8583ea9.shtml> [25.01.2018].

91 Unbekannte/r Autor/in: Expo Milano 2015 pubblica il suo Report Ufficiale. <http://www. expo2015.org/2018/06/07/expo-milano-2015-pubblica-il-suo-report-ufficiale/> [08.11.2018]. 
Ambivalenz - die allerdings erkannt werden muss - ein wichtiges Signal und appelliert, indem sie die Konflikte der Vergangenheit in den Fokus nimmt, auf konstruktive Weise an die kollektive Erinnerung.

\section{VI «Il nostro destino splendido di viaggianti»}

Einen wichtigen Impuls für die europäische Gegenwart liefern ihrerseits die Brüder de Chirico, die aufgrund der gnadenlosen Hybridität und Komplexität und womöglich ihrer weniger greifbaren italianità in der an nationalen Zuordnungen orientierten Öffentlichkeit geringere Beachtung fanden. Im 1919 nachträglich unter Giorgios Namen veröffentlichten Manifest Noi metafisici betonen die Grenzgänger, dass der Ruf der Sirenen in regelmäßigen Abständen ihren «destino splendido di viaggianti» wachhalte, der sie in die (geistige) Freiheit lockt. Sie beschreiben das hoffnungsvolle Bild ihrer geöffneten Fenster und das sich an der Sonne orientierende Bewusstsein eines neuen Morgens, bereits von Homer verheißen («albe omeriche», «tramonti incinti di domani»). In ihren auf die Weite des Meeres ausgerichteten Wohnräumen nehmen sie mit großer Offenheit Zugvögel auf, die von weit her, aus Afrika oder Amerika nach Europa gelangen und trotz ihrer physischen Erschöpfung und ihrer Fremdheit entscheidend zur kreativen Erneuerung und einer besseren Zukunft beitragen:

L'ululo delle sirene richiamatrici ci rammenta a ore fisse il nostro destino splendido di viaggianti. Nelle nostre camere vengono a cadere sfiniti dopo i lunghi voli sui mari gli uccelli sconosciuti di lontane regioni. L'Africa e l'America ci rinvigoriscono di nuovi soffi e tendono telai nel nostro animo in agguato. ${ }^{92}$

Entgegen der Schwere des futuristischen Technikkults strebt Alberto Savinio nach der Leichtigkeit des Geistes, die seinen nach einer europäischen Kultur trachtenden Höhenflug bestimmt. Sein Vorbild für die Zukunft sind die Vögel ebenso wie der beflügelte Götterbote Hermes/Mercurio, die aufgrund ihrer Schwerelosigkeit in einer todbringenden Welt überleben können, «ils surnagent». ${ }^{93}$ Beispielhaft führt den Flug in die Freiheit der kleine, aus dem Nest gefallene Spatz Leonida in

92 Giorgio de Chirico: Noi metafisici. In: Ders.: Il meccanismo del pensiero. Herausgegeben von Maurizio Fagiolo. Turin: Einaudi ( $\left.{ }^{1} 1919\right)$ 1985, S. 66-71, hier S. 68.

93 Vgl. Edoardo Costadura: Nascita dello scrittore-uccello, S. 458. Costadura führt Savinios Pseudonym auf «Savinien» als den Namen Cyrano de Bergeracs (1619-55) zurück, der in Rostands bekanntem Stück Autor von L'Autre Monde ist, das aus dem 17. Jahrhundert heraus zwei sehr zukunftsgewandte imaginäre Reisen entwirft: Les Etats et Empires de la Lune und Les Etats et Empires du Soleil. Letztere imaginäre Reise wird, Costadura zufolge, von Savinio immer wieder 
der Tragedia dell'infanzia vor, dessen Name an den König von Sparta und dessen Vernichtung in der Schlacht bei den Thermopylen erinnert: ${ }^{94}$

Più piccolo di me ma più esperto, Leonida traversò la finestra, puntò diritto nel cielo, si ridusse a una pillola bruna, e d'un tratto, come un'ombra che svanisce, sparì nella limpida atmosfera del mattino. ${ }^{95}$

\section{Literaturverzeichnis}

Aragon, Louis: Une vague de rêves. In: Ders.: CEuvres poétiques complètes, Bd. 1. Herausgegeben von Olivier Barbarant. Paris: Gallimard 2007.

Aragon, Louis: Le paysan de Paris. Paris: Gallimard ('1926) 1953.

Ballerini, Luigi: Introduzione. In: Filippo Tommaso Marinetti: Mafarka il futurista. Mailand: Mondadori 2003, S. VII-XLVIII.

Ben-Ghiat, Ruth: Fascist Modernities: Italy 1922-1945. Berkeley: University of California Press 2001.

Benn, Gottfried: Probleme der Lyrik. In: Ders.: Gesammelte Werke in vier Bänden. Bd. 1. Wiesbaden: Limes 1966, S. 494-532.

Bernard, Émile: À Marinetti. In: Poesia IV, 6 (Juli 1908).

Bianciotti, Héctor: Introduzione. In: Alberto Savinio: Souvenirs. Palermo: Sellerio (11976) 1989.

Bischof, Rita: Teleskopagen, wahlweise. Der literarische Surrealismus und das Bild. Frankfurt a. M.: Vittorio Klostermann 2001, S. 75-76.

Blumenkranz-Onimus, Noëmi: Le pré-surréalisme des futuristes italiens. In: Sandro Briosi/Henk Hillenaar (Hg.): Vitalité et contradictions de l'avantgarde. Italie-France 1909-1924. Paris: José Corti 1988, S. 27-37.

Breton, André: Introduction au Discours sur le peu de réalité. In : Ders.: Euvres complètes. Bd. 2. Herausgegeben von Marguerite Bonnet. Paris: Gallimard 1992, S. 265-280.

Breton, André: Anthologie de l'humour noir. In: Ders.: Euvres complètes. Bd. 2. Herausgegeben von Marguerite Bonnet. Paris: Gallimard 1992, S. 1122-1126.

Breton, André: Manifeste du surréalisme. In: Ders.: FEuvres complètes. Bd. 1. Herausgegeben von Marguerite Bonnet. Paris: Gallimard 1988, S. 307-346.

Breton, André: Nadja. In: Ders.: CFuvres complètes. Bd. 1. Herausgegeben von Marguerite Bonnet. Paris: Gallimard 1988, S. 748.

de Chirico, Giorgio: Noi metafisici. In: Giorgio de Chirico: /l meccanismo del pensiero. Herausgegeben von Maurizio Fagiolo. Turin: Einaudi (1919) 1985, S. 66-71.

zitiert, und zwar im Hinblick auf die Begegnung mit dem «popolo degli uccelli». Sich selbst stellt der Künstler $1936 \mathrm{im}$ Autoritratto a forma di gufo mit dem Kopf eines Uhus dar.

94 Vgl. zur Bedeutung der Erinnerung («Memoria») im Kontext der Reise Marco Sabbatini: L'argonauta, l'anatomico, il funambolo, insbesondere das Kapitel «Il mondo a volo d'uccello», S. 57-60.

95 Alberto Savinio: Tragedia dell'infanzia, S. 30. 
Costadura, Edoardo: Nascita dello scrittore uccello. In: Sergio Luzzatto/Gabriele Pedullà u.a. (Hg.): Atlante della letteratura italiana. Bd. III: Dal romanticismo a oggi. Turin: Einaudi 2012, S. 454-459.

Fähnders, Walter: Avantgarde und Moderne 1890-1933. Stuttgart: J.B. Metzler 1998.

Fontanella, Luigi: Il surrealismo italiano. Ricerche e letture. Rom: Bulzoni 1983.

Gahl, Peter: Die Fahrt des Argonauten. Das Werk Alberto Savinios von der «scrittura metafisica» zum «surrealismo archeologico». München: Wilhelm Fink 2003.

Grewe, Andrea: Melancholie der Moderne. Studien zur Poetik Alberto Savinios. Frankfurt a. M.: Vittorio Klostermann 2001.

Holzhey, Magdalena/Gerd Roos: Giorgio de Chirico und Alberto Savinio. Eine Biografie der Dioskuren. In: Paolo Baldacci/Wieland Schmied (Hg.): Die andere Moderne. De Chirico Savinio. Ostfildern/Berlin: Hatje Cantz 2001, S. 25-43.

Italia, Paola: Il pellegrino appassionato. Savinio scrittore 1915-1925. Palermo: Sellerio 2004.

Italia, Paola: Savinio, Soffici e la politica culturale del fascismo nei primi anni Venti: «Il Nuovo Paese» e il «Corriere Italiano». In: Nuova Rivista di letteratura italiana (2000), S. 389-450.

Jannini, Pasquale A.: Introduzione. In: Filippo Tommaso Marinetti: Scritti francesi. Bd. 1. Mailand: Mondadori 1983, S. 7-30.

Jemma, Rossana: La réception immédiate du Manifeste de fondation du Futurisme de Marinetti en France. In: Mariella Colin: Lettres italiennes en France. Caen: Presses Universitaires de Caen 2005, S. 145-161.

La Rosa: Leopardi, Marinetti, Futurismo e futurismi, Un rapporto infinito. In: Barbara Kuhn/ Michael Schwarze (Hg.): Leopardis Bilder. Immagini e immaginazione oder: Reflexionen von Bild und Bildlichkeit. Tübingen: Narr Francke Attempto 2019, S. 171-185.

Lautréamont: Chants de Maldoror. Chant sixième. I [3]. In : Ders: Germain Nouveau. Euuvres complètes. Paris: Gallimard 1970, S. 224-225.

Leopardi, Giacomo: All'Italia. In: Ders: Canti. Herausgegeben von Niccolò Gallo/Cesare Garboli. Turin: Einaudi (11962) 1993, S. 3-10.

Lipphardt, Anna: Der Nomade als Theoriefigur, empirische Anrufung und Lifestyle-Emblem. Auf Spurensuche im Globalen Norden. In: APuZ 26/27 (2015). <http://www.bpb.de/apuz/ 208257/der-nomade-als-theoriefigur-empirische-anrufung-und-lifestyle-emblem-auf-spurensuche-im-globalen-norden? $\mathrm{p}=$ all> [25.01.2018].

Lista, Giovanni: Marinetti et le surréalisme. In: Surréalisme/Surrealismo, Quaderni del Novecento Francese 2 (1974), S. 121-149.

Marinetti, Filippo Tommaso: Teoria e invenzione futurista. Herausgegeben von Luciano de Maria. Mailand: Mondadori 1968.

Marinetti, Filippo Tommaso: Scatole d'amore in conserva. Rom: Edizione d'Arte Fauno 1927.

Marinetti, Filippo Tommaso: Manifesto tecnico della letteratura futurista. In: Ders.: Teoria e invenzione futurista, S. 46-54.

Marinetti, Filippo Tommaso: Fondazione e Manifesto del Futurismo. In: ders.: Teoria e invenzione futurista, S. 7-13.

Meineke, Eva-Tabea: «[C]e chèvrefeuille tremblant». Der französische Surrealismus und das Mittelalter. In: Anna Isabell Wörsdörfer/Kirsten Dickhaut u. a. (Hg.): Sur les chemins de l'amititié. Beiträge zur französischen Literaturgeschichte. Freundesgabe für Dietmar Rieger. Wiesbaden: Harrassowitz 2017, S. 113-124.

Meineke, Eva-Tabea/Gesa zur Nieden: Kunst und Krieg bei Alberto Savinio. In: Zibaldone 57 (2014), S. 19-32. 
Meineke, Eva-Tabea: Rivieras de l'irréel. Surrealismen in Italien und Frankreich. Würzburg: Königshausen \& Neumann 2019.

Minella, Massimo: Il sogno dell'ingegnere che disegnò le ali alla bellezza di Asmara. <https:// ricerca.repubblica.it/repubblica/archivio/repubblica/2017/07/17/il-sogno-dellingegnereche-disegno-le-ali-alla-bellezza-di-asmara21.html > [25.01.2018].

Nelting, David/Isabel von Ehrlich: Phaëton. In: Maria Moog-Grünewald (Hg.): Mythenrezeption: die antike Mythologie in Literatur, Musik und Kunst von den Anfängen bis zur Gegenwart. Stuttgart: J.B. Metzler 2008, S. 571-577.

Paglia, Luigi: Filippo Tommaso Marinetti. In: Dizionario Biografico degli Italiani, Volume 70 (2008). <http://www.treccani.it/enciclopedia/filippo-tommaso-marinetti_(Dizionario-Biografico)/> [26.01.2018].

Sabbatini, Marco: L'argonauta, l'anatomico, il funambolo. Alberto Savinio dai Chants de la mimort a Hermaphrodito, Rom: Salerno 1997.

Savinio, Alberto: Tragedia dell'infanzia. Mailand: Adelphi 2001.

Schrader, Sabine: Einleitung. In: Sabine Schrader/Barbara Tasser (Hg.): Futurismo al $100 \%-100$ \% Futurismus. Innsbruck: Innsbruck University Press 2012, S. 11-16.

Sica, Beatrice: La poesia italiana e il surrealismo. In: Dies.: Poesia surrealista italiana. Genua: San Marco dei Giustiniani 2007, S. 9-49.

Silva, Umberto: Kunst und Ideologie des Faschismus. Frankfurt a. M.: S. Fischer 1975.

Talbot, George: Censorship in Fascist Italy 1922-43. Basingstoke: Palgrave Macmillan 2007.

Tiller, Elisabeth: Futuristische Männlichkeiten. In: Zibaldone 57 (2014), S. 77-90.

Unbekannte/r Autor/in: Expo, dopo 90 anni in mostra il «Genio futurista» di Balla. <http:// milano.corriere.it/notizie/cronaca/15_marzo_02/expo-in-mostra-genio-futurista-balla5e961592-c0f3-11e4-b2c9-4738a8583ea9.shtml> [25.01.2018].

Unbekannte/r Autor/in: Asmara, eine modernistische afrikanische Stadt. <https://www.unesco. de/kultur-und-natur/welterbe/welterbe-weltweit/asmara-eine-modernistische-stadt-afrikas-neue> [29.10.2018].

Volgger, Peter/Stefan Graf (Hg.): Architecture in Asmara. Colonial Origin and Postcolonial Experiences. Berlin: DOM publishers 2017.

Volgger, Peter/Stefan Graf (Hg.): Asmara - The Sleeping Beauty. Eine Ausstellung kuratiert von Stefan Graf und Peter Volgger. <https://aut.cc/ausstellungen/asmara-the-sleeping-beauty> [29.10.2018].

Unbekannte/r Autor/in: Expo Milano 2015 pubblica il suo Report Ufficiale. <http://www.expo2015.org/2018/06/07/expo-milano-2015-pubblica-il-suo-report-ufficiale/> [08.11.2018]. 